\title{
Using a software as a service program in sales- marketing: a case study on Odoo
}

\author{
Ana Maria Mihaela Iordache $^{1 *}$, Cezar Octavian Mihalcescu², and Beatrice Sion $^{3}$ \\ ${ }^{1}$ Romanian-American University, Department of IT, Statistics and Mathematics. No.1B Expoziţiei \\ Bld, Bucharest, Romania \\ ${ }^{2}$ Romanian-American University, Department of IT, Statistics and Mathematics. No.1B Expoziției \\ Bld, Bucharest, Romania \\ ${ }^{3}$ Romanian-American University, Department of IT, Statistics and Mathematics, No.1B Expoziției \\ Bld, Bucharest, Romania
}

\begin{abstract}
With the development of technology, implicitly of the fast and easy access to information, the requirements and exigencies of the customers have changed. The customer asks for much more information about a product before deciding to buy it, and a prompt response from the sales team will tip the balance decisively in favor of the seller. The success of an efficient sales team is represented by addressing to the right customers at the right time and in the right way. In the paper we analyzed how the Odoo program can be implemented within the sales-marketing department. Using specific modules, we followed the information flow of the marketing process, from market prospecting and to the registration in accounting of the invoices resulting from the orders made. We also presented the way in which marketing campaigns can be carried out, depending on the target group to which it is addressed.
\end{abstract}

Key words: CRM, Saas, marketing, company, Odoo, service

\section{Introduction}

In the last period the online environment has become increasingly dynamic and vast and has grown in complexity a lot. Internet communication can be done extremely easily, no longer geographical and temporary barriers, the only ones left are those of a cultural nature. Starting with 2020, with the onset of the covid-19 pandemic, the number of sales transactions on the Internet has increased at a fast pace, the companies having to adapt their main activity and, implicitly, the sales activity to the new reality.

Most of the time, the staff in the sales department is the only contact of the company with the consumers, who provide information about the products to the customers, identifying themselves unconditionally with the respective company. The activities of the sales agents include: identifying sales opportunities and potential customers, transmitting product information, negotiating sales contracts, concluding sales contracts, providing customer support in solving problems related to the sales process, conducting market research.

\footnotetext{
*Corresponding author: iordache.ana.maria.mihaela@profesor.rau.ro
} 
In order to facilitate the activity of the sales department and to have a better control from the management of an organization over the portfolio of contracts and clients, specialized software programs called Customer Relationship Management (CRM) were created. The advantages of using such a program are multiple, including: creating a single database with all customers accessible via the Internet from anywhere in the world, the existence of all customers in one place, reducing the time to process customer information, so increasing the productivity of employees' work, automating processes and eliminating repetitive activities, customer loyalty to the company's products.

Thus, when an employee in the sales department comes into contact with a customer, he notes in the program information about the potential customer, but also what he discussed with him, and at a later discussion access to information will be achieved almost instantly. In this way the customer has the satisfaction that he is important in front of the seller, he gains time in the sales process, and the risk of a human error is almost non-existent. In the conditions of a fierce competition on the market, every error on the part of the companies in the relation with the clients, the employees, respectively with the suppliers will have as a result the deterioration of the image, the decrease of the credibility and in time to its disappearance from the market.

The CRM program integrates in a single application everything a company needs to implement effective marketing strategies and increase sales, customer satisfaction, but also employee satisfaction, because an organized and professional environment is sought by all parties involved. From the point of view of a company's management, through a CRM program it is very easy to obtain real-time control over the sales activity, and the risk of losing information about customers if an employee is unable to work is much reduced.

In fact, at the end of a set period: a month, a quarter, half a year, a calendar or accounting year, the company can obtain summary reports on the situation in the sales department and, by calling the business intelligence modules included in the program, can make projections for the future. Also, depending on the criteria established for these projections, aggregate indicators can be calculated and which will further help in making strategic decisions at company level.

\section{Literature review}

In a sales system, whose complexity is increasing day by day, the market prospecting function is becoming more and more important. Scallop V.A. and Claro D.P. showed that market prospecting often depends to a large extent on how to combine three components: the team, salesperson competence and software used for market prospecting. [1] The means and the way of transmitting the information about the products of a company depend to a greater extent on the abilities of the persons from the sales team. Thus, it can be stated that the marketing process is at least as important as the process of making a product, and in order for this process to take place in optimal conditions, certain strategies have been developed. The ways in which customers are informed about a certain product are among the most diverse. Given the fact that with the development of the internet, the social media has developed a lot too, salespersons have turned their attention to it to promote their products. So, according to Larimo et el, there are four types of social marketing strategies, namely: "social commerce strategy, social content strategy, social monitoring strategy, and social CRM strategy". This classification takes into account the maturity of these strategies. [2]

Hashem N. Tareq draws attention to the fact that the level of development of the Internet in a country, in his study was referring to Jordan, entails the need to develop specialized software to gather information about customers. Thus, the Internet of Things (IoT) becomes an integral part of the market prospecting process and implicitly of the sales strategy. [3] 
Customer Relationship Management (CRM) products help to organize the marketing activity in optimal conditions. For the most part, all CRM products have approximately the same functions, but they are differentiated by elements that are very much related to the specifics of the company that uses them. Thus, there are desktop products and software as a service (SaaS) products that work mainly using the Internet. For SaaS type products, the connection to the application depends on the internet connection of the owned device.

According to Terminanto et al, the use of CRM programs and, in particular, Odoo's in the sales department, has led to the acceleration of "the process of making the offer", to higher sales, thus increasing the efficiency of the activity. [4] So, it can be said that "adopting a CRM tool may mean a competitive advantage over the competition". [5] Another study made by Suryo et al on the effects of implementing Odoo on Mc Cake showed that important advantages can be obtained, such as: "speed, accuracy, and relatively lower costs" compared to using traditional methods. [6] Looking at the field of clothing sales, Ristyawati et al concluded that the implementation of Odoo led to the efficient use of sales resources and thus to increasing the profit of the company that adopted it. [8]

Odoo can be used in various fields of activity, the most suitable companies for this being the small and medium ones, with a maximum of 250 employees. Also, being easy to use, quite adaptable to the needs of companies, but also less expensive, flexible, full control and efficient, it can be used successfully in universities too. In this way, the student will focus on the flow of information rather than on the use and implementation of the software. [7]

\section{Modeling the sales process with Odoo}

The activities of market prospecting, identifying opportunities and managing the entire chain of tasks from the contact of potential customers to the firm conclusion of a contract are very important in the Sales department of any company. The automation of the activities in this chain has as an immediate consequence the increase of the employees' productivity, in the sense that they can save time for solving the problems. Another consequence is increased sales and prompt response to customer needs.

In order to efficiently manage the customer relationship, the Odoo program offers companies several modules, namely: Discuss, Calendar, Contacts, CRM, Sales and Accounting. Each of these modules has a well-established role, as follows: Discuss allows direct contact between the sales department representative and the company's customers (potential or already existing); Calendar allows the user to set up meetings with clients in the calendar for better time management; Contacts retains information about the contacts held by the employee of the marketing department with potential customers; Sales has as main object the activity of selling the products; the Accounting module allows the registration in the accounting journals of the sales operations performed.

In addition to the modules listed above, in Odoo there are other modules related to the marketing function, modules that allow a better management of the activity by attracting potential customers on the market, namely: Email Marketing, Events, Social Marketing, Appointments, Surveys and SMS Marketing. Depending on the channels used by the company and its interest, one or more of these marketing tools can be used.

The CRM module is the main module that deals with customer relationship management, attracting new sales opportunities and transforming them into firm orders. This module has the following menu:

- Sales: my pipeline, my activities, my quotations, teams, customers

- Reporting: Pipeline and Activities

- Configuration: Settings, Sales Teams, Activity Types, Pipeline (Tags and Lost Reasons) and Lead Generation. 
The first step in the market prospecting activity is the identification of potential ways (pipelines) to attract customers. If the pipeline has been identified by the person in the sales department, he can add it to the databases by pressing the Create button (fig. 1).

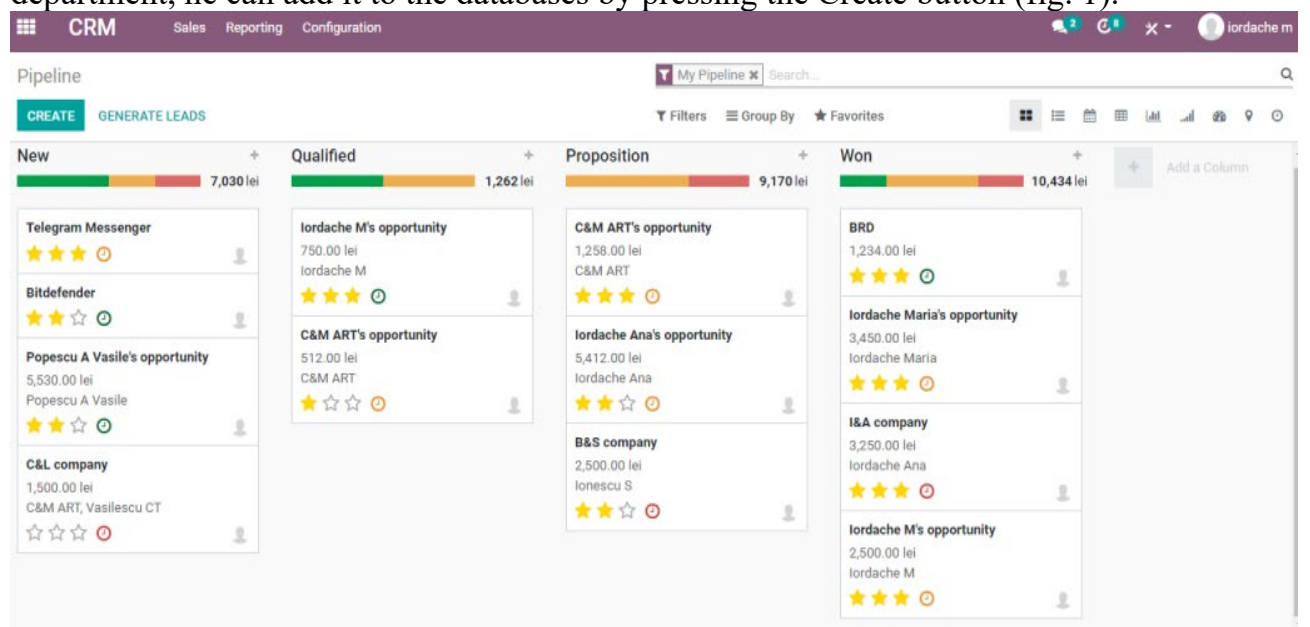

Fig. 1. Pipeline from CRM module

If, on the other hand, pipelines are not entered manually, then by pressing the generate leads button (fig. 1) the existing companies in a certain area can be selected from the Odoo database, depending on the criteria established by the sales team employee to become potential customers.

After pipelines have been identified, each company chosen as a potential customer can be assigned a priority level by selecting the stars. Thus, there are three priority levels, namely: medium (one star), high (two stars) and very high (three stars). Also, there are four stages of an opportunity, namely: new (companies are selected from the Odoo database or entered manually, they still have no contact with the seller), qualified (if there has been a contact with the potential customer and express interest), proposition (if presented to the potential client the products offered by the company) and won (if the interest of the potential client is firm, the latter requesting to be made an offer according to his requirements and further, the offer having great chances to turn into a firm order). The transition from one category to another is done by drag-and-drop.

For each company, specific activities can be established, by pressing the clock inside its rectangle, such as: email, call, meeting, to do, upload document. These activities help the person prospecting the market to better manage its potential customers. Each activity will be assigned a color: red (the activity should have been completed but not completed by the current date), yellow (the activity should be performed on the current day) and green (the activity is planned and should be completed at a later date). . If the meeting activity is selected, then the calendar will open and the time interval for it will be selected (fig. 2).

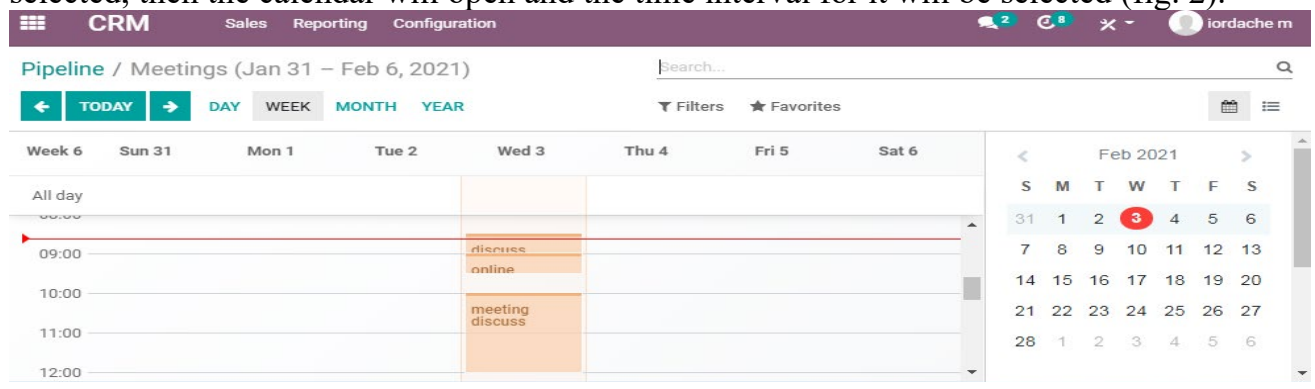

Fig. 2. Calendar for pipeline 
If we choose My Activities from the Sales menu, a window will open where all the activities established so far can be viewed (fig. 3).

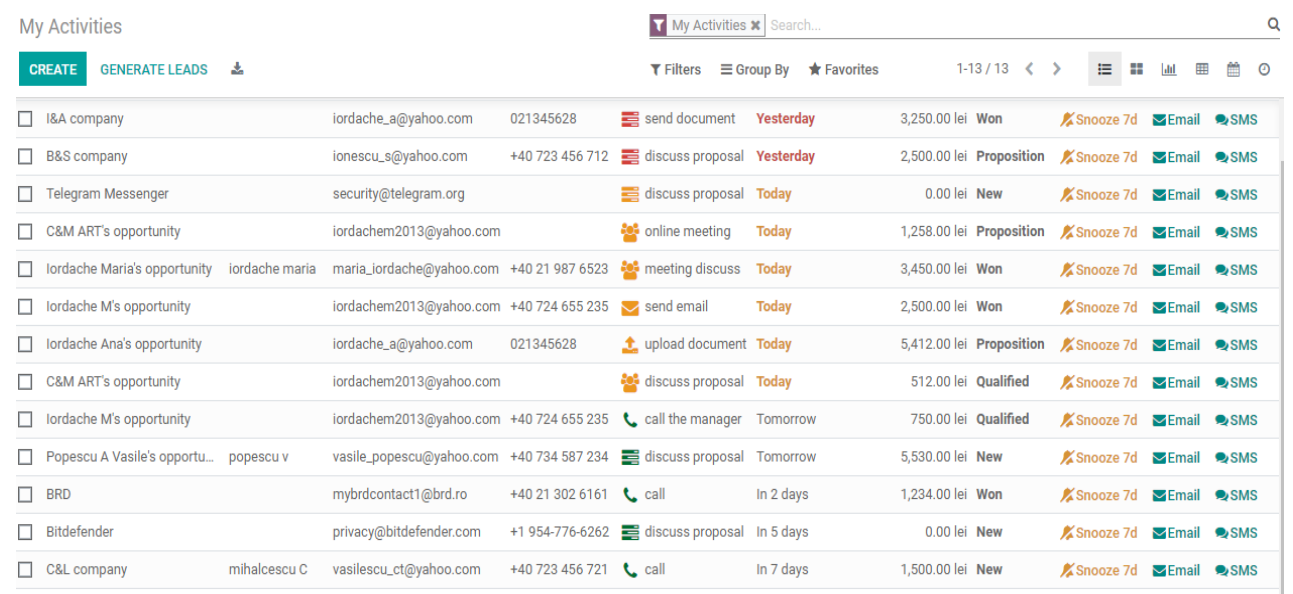

Fig. 3. My activities from Odoo CRM

An overview of all activities can be found in Fig. 3. For each opportunity the following information will be displayed: company name, contact name, contact email, phone, the next activity set by the person in the sales team, the deadline for activity, expected revenue of the activity and the stage of the opportunity (new, proposition, won and qualified). Also in this screen are integrated two other elements: email (the possibility to send an email to the company) and SMS (the service of sending SMS on the client phone).

After an opportunity has been labeled as won, we will proceed to edit the quotation and, once all the details have been established after the discussions with the client, the order is invoiced and honored.

From the Sales menu, selecting the My Quotations option opens a window in which all the offers created and their stage until that moment are visualized (fig. 4).

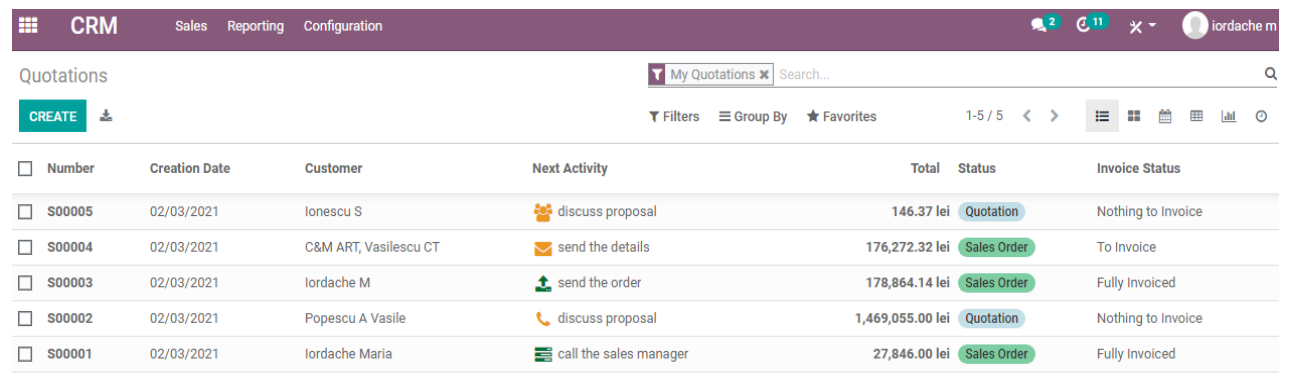

Fig. 4. The list of quotations

Thus, some offers can remain only at the offer stage, others are transformed into firm orders, orders that can be partially invoiced or are fully invoiced. For each offer is also presented the next activity, but also the value brought in the company.

To make a new offer, click the Create button and enter details regarding the customer, the product or products in the offer, other related products, the way of signing the offer, the payment deadline, the expiration date of the offer (fig. 5). 
Quotations / New

SAVE DISCARD

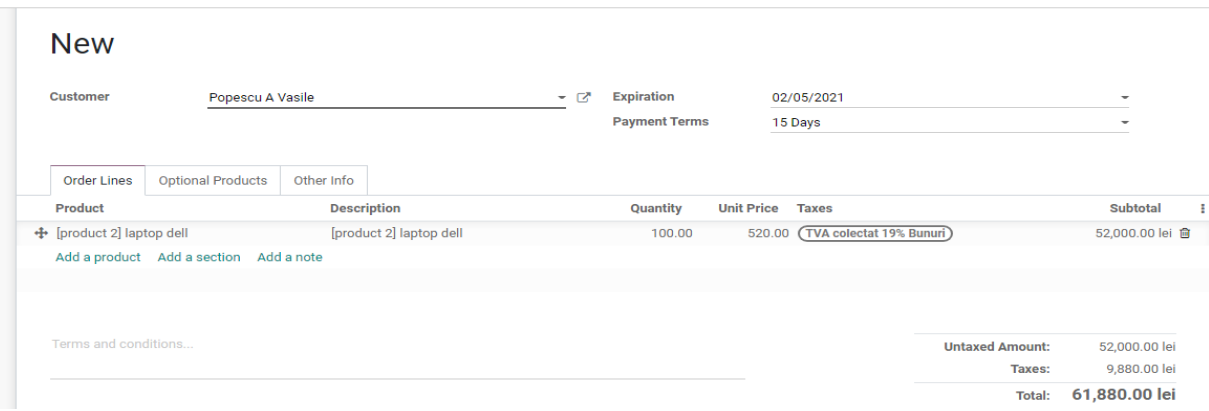

Fig. 5. Creating a new quotation

Also on the new offer we will be able to enter information about the salesperson, whether or not we can accept the online signature and payment on the internet, the delivery time of the products, but also the fiscal position of the invoice. Furthermore, the offer is sent by email. After the discussion between the potential customer and the seller and if the terms are accepted by both parties, the offer turns into a firm order by pressing the confirm button (fig. $6)$.

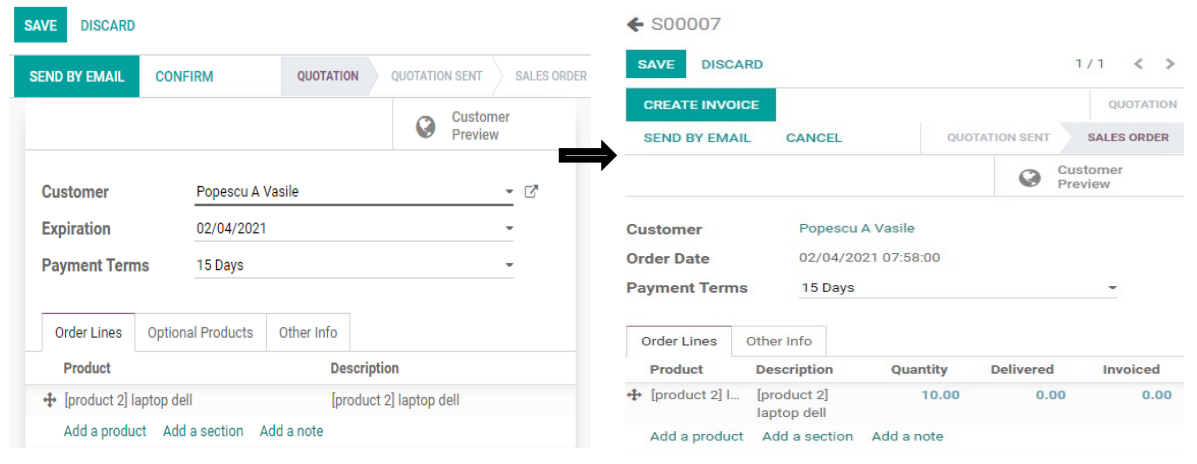

Fig. 6. From quotation to an order

Next, choose the type of invoice: regular or with down payment invoice, percentage or fixed amount and it will be created a draft invoice, which need to be validated and then registered in accounting module (fig. 7).
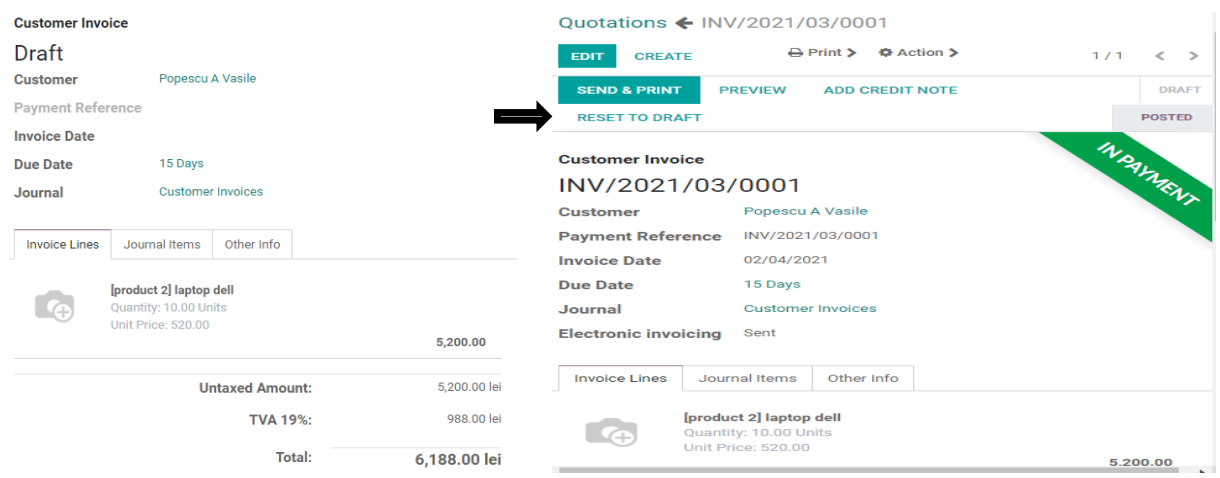

Fig. 7. From draft invoice to payment 
After viewing the draft invoice, the user has the possibility to modify if necessary certain elements and to confirm. At this moment, an invoice registration number is given and further payment is registered in the accounting by pressing the register payment button. In order to register a payment, information must be provided regarding the type of payment, cash or bank, payment method, manual or batch deposit, amount, payment date and the register number of the document which accompanies the payment (fig. 7). If you want to change certain elements of the payment registration process, press the reset to draft button and return to the order confirmation stage.

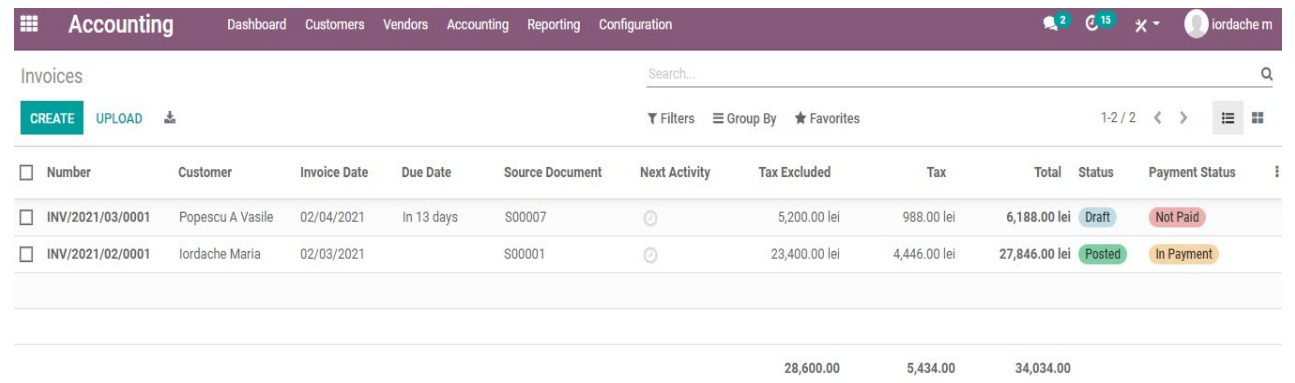

Fig. 8. Invoices from accounting module

In the accounting module (fig. 8) the transmitted invoices can be observed and must be confirmed. Also, depending on the time of sale and the established conditions, invoices can be uploaded manually.

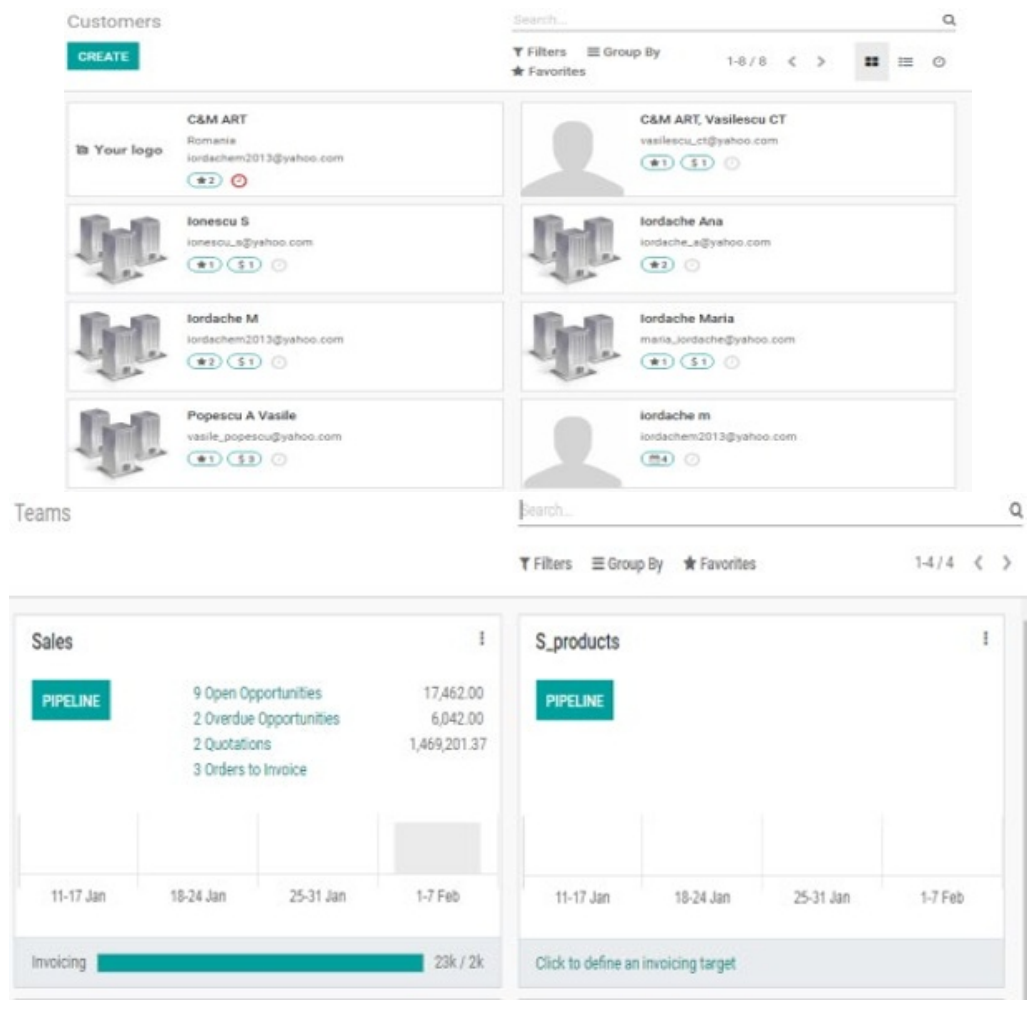

Fig. 9. Overview on customers and teams 
Returning to the CRM module, for each sales team several performance indicators can be observed, as well as the total value from a certain period of each of them, namely: number of open opportunities, number of overdue opportunities, number of quotations and the number of orders to invoice (fig. 9).

From the configuration menu in the same module, various settings can be made regarding the market prospecting activity, as follows: sales teams can be created or updated, activity types can be updated, lost reasons and pipeline tags can be defined.

From the Reporting menu we can have an overview of the activities carried out and the pipelines. Depending on the various criteria selected by the user, important information can be found to further support the decisions at the level of the sales-marketing department, but also at the level of top management. Among the criteria are: automated probability allocated depending on the stage in which a certain pipeline is located, bounce, days to assign, days to close, days to convert, exceeded closing days, expected revenue, probability given by the person of a certain team, prorated revenue and count. In our opinion, one of the most important performance criteria for the marketing department is the total amount brought by each marketing campaign, depending on the opportunity states being prospected.

In order to attract and retain customers, marketing campaigns are carried out at different times. Depending on the channel through which the information will be disseminated, Odoo has several modules: email marketing, sms marketing, appointments, events, social marketing and surveys. In each of these modules different elements are defined, depending on the type of activity carried out. To automate a marketing campaign there is the Marketing Automation module. Within this module the user can define a campaign, bringing together all the other activities defined in the marketing modules. It can also set the start time of the campaign and its duration. For each ongoing campaign, Odoo creates a workflow with each defined activity and, for each of them, there are presented the current stage and some performance indexes.

\section{Conclusion}

With the development of the need for quick access to information in all areas, programs have emerged to meet these requirements. Enterprise resources planning (ERP) programs, with the CRM component, have efficiently responded to this human development need. The Odoo program, especially the CRM module and its related modules, has successfully responded to various operations within the sales department. Although it is aimed at small and mediumsized companies, up to 250 employees, this program can be used successfully in larger companies, provided that the flow of information is at an appropriate level. The balance of benefits brought - resources spent is super-unitary, which means that Odoo can be implemented at the level of companies with quite low costs, the benefits being high, in a short time. Of course, the market for CRM programs has developed in proportion to the demand and currently there are thousands of products of this type. Each of these products tries to resist on the market by exploiting different elements: ease of use, small implementation and maintenance resources, specific field of activity of the company that adopts it, ease of integration with other existing programs within the company that buys it, anticipation and automation of activities and others. Each company, depending on the internal requirements, the offer of existing CRM products on the market and the resources held, chooses the right product in order to obtain a competitive advantage.

\section{References}

1. V.A. Vieira, D.P. Claro, D. P., Sales prospecting framework: Marketing team, salesperson competence, and sales structure, BAR, 17(4), e200025 (2021) 
2. F. Li, J. Larimo, L.C. Leonidou, Social media marketing strategy: definition, conceptualization, taxonomy, validation, and future agenda. J. of the Acad. Mark. Sci. 49, 51-70 (2021)

3. Hashem N. Tareq, The reality of internet of things (IoT) in creating a data-driven marketing opportunity: mediating role of customer relationship management (CRM), J. of Theoretical and Appl Inf Tech, 329-342, 99(2) (2021)

4. A. Terminanto, R. Hidayat, A.N. Hidayanto, Implementation of enterprise resource planning using Odoo module sales and CRM. Case study: PT Ecosains Hayati, IOP Conf. Ser.: Mater. Sci. Eng. 10th ISIEM, 277, 012034 (2017)

5. T.M. Cruz, J. Gutiérrez-Artacho, J. Bernardino, Open Source CRM Tools for Small Companies, WorldCIST 2017, Adv in Intel Syst and Comp, 569, Springer, Cham (2017)

6. D. A. Suryo, S. Abdullah, T. Siswanto, Odoo ERP implementation point of sale module at MC Cake, Intelmatics, 26-31, 1(1) (2021)

7. J.Y. Wu, L.T. Chen, Odoo ERP with Business Intelligence Tool for a Small-Medium Enterprise: A Scenario Case Study, Proceedings of the 2020 11th International Conference on E-Education, EBusiness, E-Management, and E-Learning, IC4E 2020, 323-327 (2020)

8. Ni. K. Y. Ristyawati, I. K. A. Purnawan, G. M. A. Sasmita, The Implementation of Enterprise Resource Planning (ERP) on Sales Management Module using Odoo 11, IJTSRD, 1-4, 4(4), 2020

9. Ce este un CRM available at https://www.softone.ro/ce-este-crm/

10. Online Ideea available at https://www.onlineideea.com/crm-gratuit/

11. I. Smedescu, M.P. Ratiu, C.I. Negricea, Bazele marketingului (Ed. Universitara, Bucuresti, 2008)

12. P. Kotler, G. Armstrong, Principiile marketingului editia a IV-a (Ed. Teora, Bucuresti, 2000) 